\title{
Psychosocial predictors of patient adherence to disease-modifying therapies for multiple sclerosis
}

\author{
This article was published in the following Dove Press journal: \\ Patient Preference and Adherence \\ 9 March 2017 \\ Number of times this article has been viewed
}

\author{
Fahad D Alosaimi' \\ Alaa AIMulhem² \\ Hanan AIShalan² \\ Mohammad Alqazlan ${ }^{3}$ \\ Abdulgader Aldaif ${ }^{4}$ \\ Matthew Kowgier ${ }^{5}$ \\ Janooshsheya \\ Balasundaram ${ }^{6}$ \\ Sanjeev Sockalingam ${ }^{6,7}$ \\ 'Department of Psychiatry, ${ }^{2}$ College \\ of Medicine, King Saud University, \\ ${ }^{3}$ Department of Mental Health, King \\ Faisal Specialist Hospital and Research \\ Centre, ${ }^{4}$ Department of Neurology, \\ King Saud University, Riyadh, Saudi \\ Arabia; ${ }^{5}$ Dalla Lana School of Public \\ Health, University of Toronto, ${ }^{6}$ Centre \\ for Mental Health, University Health \\ Network, ${ }^{7}$ Department of Psychiatry, \\ University of Toronto, Toronto, ON, \\ Canada
}

Objectives: Our aim was to identify the impact of psychosocial predictors, specifically relationship style, depressive symptoms, anxiety symptoms, cognitive impairment, and culturespecific disease beliefs, on treatment adherence for multiple sclerosis (MS) patients.

Methods: In this cross-sectional observational study, patients from two MS clinics in Saudi Arabia completed self-reported questionnaires focused on MS treatment adherence, physical symptom burden, relationship style, cultural beliefs, depressive symptoms, anxiety, and cognitive impairment.

Results: A total of $163 \mathrm{MS}$ patients participated, $81.6 \%$ of them were female, and the mean age of the patients was 31.6 years. Mean patient-reported adherence to their MS treatment regimen was $79.47 \% \pm 25.26 \%$. Multivariate linear regression analysis only identified patients' belief that their MS was due to "supernatural" forces as being significantly negatively associated with MS medication adherence.

Conclusion: This study demonstrates the importance of cultural interpretations to MS medication adherence in comparison to psychosocial factors. Education and family involvement in the treatment planning may address this issue and warrant further research.

Keywords: multiple sclerosis, adherence, depression, attachment style, culture

\section{Introduction}

Approximately 2 million people worldwide are affected by multiple sclerosis (MS), a chronic and recurrent inflammatory, demyelinating, and neurodegenerative disease of the central nervous system. ${ }^{1}$ MS is the leading cause of nontraumatic injury in young adults, and the life expectancy for patients with MS ranges from 7 to 14 years less than that of the general population. ${ }^{2-6}$

Treatment of MS consists of treatment of acute relapses, prevention of relapses, wellness programs, treatment of comorbidities, and delaying disease worsening through the use of disease-modifying treatments (DMTs) such as immunomodulators and immunosuppressants. ${ }^{7}$ Despite clinical benefits and reductions in hospitalization with DMTs, patients with MS have had difficulty adhering to these treatment regimens. In a narrative review of 24 studies, Menzin et $\mathrm{al}^{8}$ showed that adherence rates with DMTs ranged from $41 \%$ to $88 \%$, with higher rates noted in prospective studies. Several factors have been found to contribute to reduced patient adherence to MS treatment with DMTs, including more frequent dosing regimen of DMTs, anxiety, depression, cognitive impairment, medication adverse effects, and patients' belief that treatment will not be efficacious. ${ }^{8-14}$ Moreover, there is a geographic variability with treatment outcomes, with notably lower MS discontinuation rates in countries such as Spain and Italy. ${ }^{15}$
Correspondence: Sanjeev Sockalingam

Toronto General Hospital,

200 Elizabeth Street-8EN-228,

Toronto, ON M5G 2C4, Canada

$\mathrm{Tel}+\mathrm{I} 4163403762$

Fax +14163404198

Email sanjeev.sockalingam@uhn.ca 
In addition to depression, anxiety, and cognitive difficulties, limited research has explored additional psychological variables impacting adherence. One variable is attachment style, which posits that the earliest bonds of children with their parents continue to impact social relationships, including health care relationships, and generate persistent patterns of interpersonal interaction as adults, which are fairly static and highly stable over time. ${ }^{16}$ These models for relating with others can help explain the reaction of patients to illness events, such as the diagnosis and treatment of MS. ${ }^{17}$ For example, attachment or relationship style, specifically an avoidant relationship style, has been associated with reduced adherence to medical treatments and follow-up care. ${ }^{18-20}$ Moreover, diabetic patients with an avoidant relationship style had a higher rate of mortality 10 years later related to poor treatment adherence. ${ }^{21}$ Based on our review of the literature, there have been no studies exploring the role of avoidant attachment style as a predictor of DMT nonadherence in MS patients.

In this study, we aimed to identify psychosocial predictors of DMT treatment adherence for MS patients seen in two MS clinics in Saudi Arabia. We specifically focused on patientrelationship style, depressive symptoms, anxiety symptoms, and cognitive impairment as predictors of MS treatment adherence. Given the potential for cultural factors to influence treatment adherence, we also aimed to study the effects of common culture-specific beliefs regarding MS in a patient population from Saudi Arabia. We hypothesized that higher anxiety symptoms, depressive symptoms, increased cognitive impairment, and greater avoidant relationship style scores would be associated with reduced medication adherence.

\section{Methods}

\section{Study sample}

We recruited consecutive patients seen in follow-up in MS clinics from two hospitals in Riyadh, Saudi Arabia: King Khalid University Hospital and King Faisal Hospital. Patients were recruited between January 2014 and June 2015. Patients were included in the study if they were $\geq 18$ years, treated with DMT or medications for MS-associated symptoms (eg, pain, spasticity, and numbness), had a neurologist-confirmed diagnosis of MS, and could speak and read Arabic. There were no exclusion criteria.

\section{Study procedures}

We used a cross-sectional study design. Patients attending the MS clinics at either of the two hospitals were introduced to the research study by clinical team members. A research assistant followed up with interested study participants and obtained informed consent. Patients who consented to participate in the study were administered a self-report study questionnaire to complete at the visit. All questionnaires were collected at the conclusion of their medical visit. Written informed consent was obtained from all individual participants included in the study.

\section{Study measures}

The study questionnaire administered to study patients included the following three sections: a sociodemographic/ cultural section, a psychiatric history section, and a medical history section. Neurologists and psychiatrists reviewed each component of the questionnaire to ensure the relevance and applicability of each question.

Demographic data collected included age, sex, education level, and employment. Within Saudi Arabia, patients' belief regarding the etiology of their illness plays an important role. Therefore, we included a question asking patients if they felt that their MS was caused by supernatural reasons such as magic, possession, and/or an "evil eye".

Medical information included duration of MS and presence of an additional chronic medical condition. This information was extracted from patients' medical records.

Self-reported treatment adherence was measured using a visual analog scale (VAS) ranging from $0 \%$ to $100 \%$ to measure treatment adherence. The VAS has been used in a validated self-report adherence scale. ${ }^{22}$ Subjects were asked to indicate their adherence to their "doctor's multiple sclerosis treatment recommendation" on the VAS.

Depressive symptoms were measured using the Arabic version of the Patient Health Questionnaire-9 (PHQ9). The PHQ9 has been used to measure depression in various medical populations, including neurological populations (reference). The PHQ9 is a 9-item scale with each item scored 0 to 3 and summed to yield a total score (range: $0-27) .{ }^{23}$ PHQ9 severity cut-off point scores are 5 for mild, 10 for moderate, 15 for moderately severe, and 20 for severe depressive symptoms.

Participants' anxiety symptoms were measured using the Arabic version of the Generalized Anxiety Disorder 7-item scale (GAD7), a 7-item tool validated in 2,740 primary care patients. ${ }^{24}$ The GAD7 severity cut-points are 5 for mild, 10 for moderate, and 15 for severe anxiety.

The Patient Health Questionnaire-15 (PHQ15) is a measure traditionally used in patients suffering from functional disorders; however, studies have utilized the PHQ-15 as a measure of physical symptoms. ${ }^{25-27}$ The PHQ15 consists 
of 15 items scored 0 (not bothered at all) to 2 (bothered a lot). Scores on the PHQ-15 range from 0 to 30 with higher scores indicating greater physical symptom burden. Physical symptoms listed on the PHQ-15 include headache, back pain, and gastrointestinal symptoms.

Relationship (attachment style) was assessed using the Experiences in Close Relationships 16-item scale (ECR16), a 16-item scale that has been validated against the longer ECR-32. ${ }^{28}$ The ECR16 yields two separate scores: a total anxious (ECR16-Anx) and a total avoidant (ECR16-Avoid) attachment score based upon scoring of eight items to each attachment style. Each attachment score ranges from 8 to 56 with higher anxious or avoidant attachment scores representing greater attachment insecurity.

Cognitive impairment was measured using the Montreal Cognitive Assessment (MOCA), a tool assessing eight domains. ${ }^{29}$ Scores on the MOCA range from 0 to 30 , with a score of 25 being a cut-point for mild cognitive impairment.

Given that there are no preexisting Arabic versions of the PHQ15 and ECR-16 scales, we developed an Arabic version of this measure based on the previously validated English versions of these measures. Both scales were first translated into Arabic by a linguistic specialist, fluent in both English and Arabic. Then, another specialist, fluent in both English and Arabic, carried out back translation into English. During this time, the back translation and the original scale were compared and any differences were discussed and resolved. The study questionnaire and all the six scales were then piloted on 20 individuals before the study began. The question and item wording were modified based on the feedback from the pilot sample prior to using these instruments in the study.

\section{Ethical approval}

The ethical approval for this study was obtained from the Institutional Review Board at Faculty of Medicine at King Saud University, Riyadh, Saudi Arabia. All procedures performed in studies involving human participants were in accordance with the ethical standards of the institutional and/ or national research committee and with the 1964 Declaration of Helsinki and its later amendments or comparable ethical standards.

\section{Statistical analysis}

Descriptive statistics for continuous variables were reported as mean \pm standard deviation (SD) and frequencies (percentages) were reported for categorical variables. To investigate predictors of treatment adherence, we used multiple linear regression analysis adjusting for age, sex, and additional factors, which showed univariate associations with treatment adherence at the $10 \%$ level of statistical significance. Potential covariates considered were MS disease duration, PHQ-9, GAD-7, comorbidities (yes/no), ECR16-Anx, ECR16-Avoid, family history of MS, belief that MS is associated with nervous psychological disorder (yes/no), belief that MS is associated with evil eye or magic or possession, previous mental illness, relationship status, education, MOCA score, and employment status. All $P$-values were two sided, and statistical significance was defined as $P<0.05$. Data were analyzed in the R statistical software (version 3.2.2).

\section{Results}

A total of $163 \mathrm{MS}$ patients participated in this research study, who represent $80 \%$ of the approached patients in the MS clinics. Participant demographics and measure scores are summarized in Table 1. Most patients were female (81.6\%), and the mean age of patients was 31.6 years.

Mean patient-reported adherence to their MS treatment regimen was $79.47 \% \pm 25.26 \%$, although the range for the VAS adherence percentage was $0 \%$ to $100 \%$. Most patients

Table I Study participant characteristics

\begin{tabular}{ll}
\hline Participant characteristics & \\
\hline Sex (female) & $133(81.6 \%)$ \\
Education (bachelor degree or higher) & $102(62.6 \%)$ \\
Relationship status (married)* & $84(51.5 \%)$ \\
Employment (employed)* & $62(38 \%)$ \\
Additional chronic medical condition & $50(30.7 \%)$ \\
Duration of MS (years) & $6.39 \pm 3.97$ \\
DMT type & \\
Interferon-beta & $122(74.8 \%)$ \\
Natalizumab & $9(5.5 \%)$ \\
Fingolimod & $6(3.7 \%)$ \\
Teriflunomide & $1(0.61 \%)$ \\
Other & $25(15.3 \%)$ \\
Family history of MS & $31(19.0 \%)$ \\
Adherence on VAS (\%) & $79.47 \pm 25.26$ \\
Supernatural belief & $85(52.1 \%)$ \\
Past history of psychiatric illness & $8(4.9 \%)$ \\
PHQ9 & $9.67 \pm 6.52$ \\
GAD7 & $8.37 \pm 5.73$ \\
PHQI5 & $10.60 \pm 5.65$ \\
ECR-I6 anxious & $33.79 \pm I 1.30$ \\
ECR-I6 avoidance & $23.57 \pm 9.54$ \\
MOCA & $20.23 \pm 4.66$ \\
\hline Notes & \\
\hline
\end{tabular}

Notes: *Data listed as frequency (\%); all other data listed as mean \pm standard deviation. Married included common law; however, no participants were in a common-law relationship. Supernatural belief represents patients' belief that MS is due to supernatural reasons like magic, possession, and/or an "evil eye".

Abbreviations: MS, multiple sclerosis; DMT, disease-modifying treatment; VAS, visual analog scale; PHQ9, Patient Health Questionnaire-9; GAD7, Generalized Anxiety Disorder 7-item scale; PHQ 15, Patient Health Questionnaire-I5; ECRI6, Experiences in Close Relationships 16-item scale; MOCA, Montreal Cognitive Assessment. 
were prescribed interferon-beta $(n=122,74.8 \%)$, followed by natalizumab $(n=9,5.5 \%)$ and fingolimod $(n=6,3.7 \%)$. No patients were treated with glatiramer acetate and 24 patients were taking other medications for MS therapies such as muscle relaxants (eg, baclofen), medications for pain (eg, amitriptyline), and steroid medications (eg, methylprednisone).

Psychological symptom scores are reported in Table 1. The mean MOCA score was 20.23 \pm 4 .66, suggestive of some cognitive impairment. Mean PHQ9 and GAD7 scores were $9.67 \pm 6.52$ and $8.37 \pm 5.73$, respectively. Approximately half $(46.6 \%)$ of the participants reported moderate or higher depressive symptoms as per the PHQ9. Approximately twothirds $(64.4 \%)$ of participants reported moderate or severe anxiety as per the GAD7. Physical symptom score on the

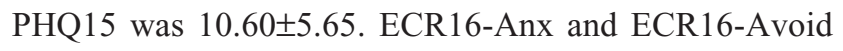
were $33.79 \pm 11.30$ and $23.57 \pm 9.54$, respectively.

\section{Variables associated with MS therapy adherence}

Univariate analysis of demographic, MS disease-specific, and psychological variables identified significant associations with specific variables (Table 2$)$. PHQ9 scores $(P=0.02)$, GAD7 scores $(P<0.01)$, ECR-Avoid $(P=0.02)$, and belief that MS was related to "supernatural reasons" $(P<0.01)$ were significantly associated with MS medication nonadherence.

Table 3 summarizes the findings on multivariate linear regression analysis. Only patients' belief that their MS was

Table 2 Univariate analysis examining relationship between variables and VAS treatment adherence

\begin{tabular}{llll}
\hline Variables & Beta & $\begin{array}{l}\text { Standard } \\
\text { error }\end{array}$ & P-value \\
\hline Age & 0.08 & 0.22 & 0.71 \\
Sex (female vs male) & -4.94 & 5.11 & 0.34 \\
Disease duration & -0.50 & 0.51 & 0.32 \\
PHQ9 & -0.71 & 0.30 & 0.02 \\
GAD7 & -1.01 & 0.32 & $<0.01$ \\
ECR-Anx & -0.33 & 0.17 & 0.06 \\
ECR-Avoid & -0.47 & 0.21 & 0.02 \\
Family history of MS & -9.31 & 5.00 & 0.06 \\
Supernatural belief & -13.92 & 3.82 & $<0.01$ \\
Past psychiatric illness & 3.84 & 9.18 & 0.68 \\
Marital status (married vs not married) & 1.31 & 3.97 & 0.74 \\
Education (bachelor or higher vs lower & -5.27 & 4.10 & 0.20 \\
than bachelor degree) & & & \\
Employment status & 5.40 & 4.07 & 0.19 \\
(unemployed vs employed) & & & \\
MOCA score & 0.06 & 0.43 & 0.89 \\
\hline
\end{tabular}

Abbreviations: VAS, visual analog scale; PHQ9, Patient Health Questionnaire-9; GAD7, Generalized Anxiety Disorder 7-item scale; ECR-Anx, Experiences in Close Relationships total anxious; ECR-Avoid, Experiences in Close Relationships total avoidant; MOCA, Montreal Cognitive Assessment; MS, multiple sclerosis.
Table 3 Multivariate regression analysis examining relationship between variables and VAS treatment adherence

\begin{tabular}{llll}
\hline Variable & Estimate & Standard error & $P$-value \\
\hline Age & 0.02 & 0.22 & 0.94 \\
Sex (female vs male) & -1.88 & 5.02 & 0.71 \\
PHQ9 & -0.51 & 0.35 & 0.15 \\
GAD7 & -0.66 & 0.44 & 0.14 \\
ECR-Anx & -0.01 & 0.20 & 0.97 \\
ECR-Avoid & -0.26 & 0.21 & 0.22 \\
Family history of MS & -9.61 & 4.92 & 0.05 \\
Supernatural belief & -11.41 & 4.00 & $<0.01$ \\
\hline
\end{tabular}

Abbreviations: VAS, visual analog scale; PHQ9, Patient Health Questionnaire-9; GAD7, Generalized Anxiety Disorder 7-item scale; ECR-Anx, Experiences in Close Relationships total anxious; ECR-Avoid, Experiences in Close Relationships total avoidant; MS, multiple sclerosis.

due to supernatural reasons was significantly negatively associated with MS medication adherence. Family history of MS showed a nominal association with poorer MS medication adherence $(P=0.05)$. No other predictor variables in the linear regression model were significant.

\section{Discussion}

In this study, we aimed to identify psychological factors influencing MS medication adherence in a patient sample from MS Clinics in Saudi Arabia. Our study is the first to include relationship style as a variable influencing MS treatment adherence. The results from this study failed to show any relationship between relationship (attachment) style and medication adherence in MS patients. Our finding that avoidant relationship style was not a significant predictor contrasts previous studies demonstrating poor treatment adherence in diabetes and hepatitis patient populations. ${ }^{18,20}$ Moreover, depression and anxiety symptoms also did not significantly predict self-reported MS treatment adherence in our multivariate regression model. Our results contrast with previous data showing that MS patients with depression are half as likely to be adherent with MS medications. ${ }^{30}$ In fact, only patients' belief that their MS was due to the supernatural reasons was a significant predictor of poorer treatment adherence.

It is possible that failure to replicate findings in psychosocial domains may be related to the use of these instruments in a patient sample from the middle east. An Arabic version of the ECR16 has not been validated, and although we were thoughtful in the process of our translation, the ECR16 may have been unable to measure this construct accurately in this Arabic-speaking patient sample. However, we were able to use Arabic versions of the PHQ9 and GAD7 and were still unable to find a significant relationship between 
these variables and VAS adherence scores. The potential for geographic differences in the role of relationship style on medical treatment adherence should be explored in future studies, as most of the initial work has been limited to North America.

In addition, we found that patient perception of illness was a significant predictor for MS medication adherence. When "nonmedical forces" such as magic, possession, and/or an evil eye are a common explanation for medical ailments, treatment with biomedical interventions may be seen as contradictory to the patient's belief system. Moreover, data from a meta-analysis confirm that patients who perceive greater disease severity threat are more likely to be adherence to treatment. ${ }^{31}$ However, patients who believe that nonmedical forces are the cause of MS are less likely to perceive disease severity threat and have hope that these forces, such as magic or the evil eye, can be reversed. Therefore, our findings highlight the need to consider cultural beliefs in most research and clinical care exploring MS treatment adherence.

Limitations of our study include reliance on a self-report measure for medication nonadherence. Given this was the first study in this region examining medication nonadherence in MS, we used a validated scale that was used previously in patients with a chronic medical condition. ${ }^{18}$ As mentioned, two scales, namely the PHQ15 and the ECR16, were not previously validated for use in an Arabic-speaking patient population, which could have limited our results. We were unable to replicate previous psychosocial findings and this may be due to a lack of study power or possibly due to patient complacency. In addition, our study was conducted in one cultural context, which could limit generalizability; however, we see this as a strength given the abovestated cultural influences that have not been previously noted in MS studies. Moreover, our male-to-female distribution reflects the distribution of MS in other samples. ${ }^{32}$ Finally, our study was unable to comment on the relationship between selfreported medication nonadherence and clinical outcomes in this patient sample. This warrants further exploration in future studies.

\section{Conclusion}

Although we failed to replicate previous relationships between medical treatment adherence and specific psychological factors, our study identified a significant association between cultural beliefs about MS and MS treatment nonadherence in a sample of patients from Saudi Arabia. The study reinforces the need to consider cultural interpretations of MS as a factor contributing medication adherence and the need for replicating studies on MS treatment adherence across a range of geographic settings. Education and family involvement in the treatment planning may assist with adherence and warrant further exploration in these settings.

\section{Acknowledgments}

We would like to express our gratitude to Dr Ibrahim Alquraishi and Ms Ahoud Alluhaim for their assistance in preparation of study tools and Ms Fatima Jama for her help in data entry. Moreover, we would also like to thank Dr Mohammad Zuheir Alkawi (Senior Consultant, Neurology Department, King Faisal Specialist Hospital and Research Centre, Riyadh, Saudi Arabia) for his administrative support. Funding for this study was provided by Deanship of Scientific Research at King Saud University, Riyadh, Saudi Arabia, research group no RG-1435-087.

\section{Disclosure}

The authors report no conflicts of interest in this work.

\section{References}

1. World Health Organization: Atlas multiple sclerosis resources in the world 2008. Geneva: WHO Press; 2008.

2. Compston A, Coles A. Multiple sclerosis. Lancet. 2008;372(9648): $1502-1517$.

3. Noseworthy JH, Lucchinetti C, Rodriguez M, Weinshenker BG. Multiple sclerosis. N Engl J Med. 2000;343(13):938-952.

4. Weinshenker BG, Bass B, Rice GP, et al. The natural history of multiple sclerosis: a geographically based study. I. Clinical course and disability. Brain. 1989;112(pt 1):133-146.

5. Sadovnick AD, Ebers GC, Wilson RW, Paty DW. Life expectancy in patients attending multiple sclerosis clinics. Neurology. 1992;42(5): 991-994.

6. Scalfari A, Knappertz V, Cutter G, Goodin DS, Ashton R, Ebers GC Mortality in patients with multiple sclerosis. Neurology. 2013;81(2): 184-192.

7. Goodin DS, Frohman EM, Garmany GP Jr, et al. Disease modifying therapies in multiple sclerosis: report of the Therapeutics and Technology Assessment Subcommittee of the American Academy of Neurology and the MS Council for Clinical Practice Guidelines. Neurology. 2002;58(2):169-178.

8. Menzin J, Caon C, Nichols C, White LA, Friedman M, Pill MW. Narrative review of the literature on adherence to disease-modifying therapies among patients with multiple sclerosis. J Manag Care Pharm. 2013; 19(1 suppl A):S24-S40.

9. Turner AP, Williams RM, Sloan AP, Haselkorn JK. Injection anxiety remains a long-term barrier to medication adherence in multiple sclerosis. Rehabil Psychol. 2009;54(1):116-121.

10. DiMatteo MR, Lepper HS, Croghan TW. Depression is a risk factor for noncompliance with medical treatment: meta-analysis of the effects of anxiety and depression on patient adherence. Arch Intern Med. 2000;160(14):2101-2107.

11. Bruce JM, Hancock LM, Arnett P, Lynch S. Treatment adherence in multiple sclerosis: association with emotional status, personality, and cognition. J Behav Med. 2010;33(3):219-227.

12. Beer K, Muller M, Hew-Winzeler AM, et al. The prevalence of injection-site reactions with disease-modifying therapies and their effect on adherence in patients with multiple sclerosis: an observational study. BMC Neurol. 2011;11:144. 
13. Steinberg SC, Faris RJ, Chang CF, Chan A, Tankersley MA. Impact of adherence to interferons in the treatment of multiple sclerosis: a non-experimental, retrospective, cohort study. Clin Drug Investig. 2010;30(2):89-100.

14. Treadaway K, Cutter G, Salter A, et al. Factors that influence adherence with disease-modifying therapy in MS. J Neurol. 2009;256(4): $568-576$.

15. Meyniel C, Spelman T, Jokubaitis VG, et al. Country, sex, EDSS change and therapy choice independently predict treatment discontinuation in multiple sclerosis and clinically isolated syndrome. PLoS One. 2012;7(6): e38661.

16. Bowlby J. Attachment and Loss. New York, NY: Basic Books; 1969.

17. Hunter JJ, Maunder RG. Using attachment theory to understand illness behavior. Gen Hosp Psychiatry. 2001;23(4):177-182.

18. Sockalingam S, Blank D, Abdelhamid N, Abbey SE, Hirschfield GM. Identifying opportunities to improve management of autoimmune hepatitis: evaluation of drug adherence and psychosocial factors. J Hepatol. 2012;57(6):1299-1304.

19. Sockalingam S, Cassin S, Hawa R, et al. Predictors of post-bariatric surgery appointment attendance: the role of relationship style. Obes Surg. 2013; 23(12):2026-2032.

20. Ciechanowski P, Russo J, Katon W, et al. Influence of patient attachment style on self-care and outcomes in diabetes. Psychosom Med. 2004; 66(5):720-728.

21. Ciechanowski P, Russo J, Katon WJ, et al. Relationship styles and mortality in patients with diabetes. Diabetes Care. 2010;33(3):539-544.

22. Svarstad BL, Chewning BA, Sleath BL, Claesson C. The Brief Medication Questionnaire: a tool for screening patient adherence and barriers to adherence. Patient Educ Couns. 1999;37(2):113-124.

23. Kroenke K, Spitzer RL, Williams JB. The PHQ-9: validity of a brief depression severity measure. J Gen Intern Med. 2001;16:606-613.
24. Spitzer RL, Kroenke K, Williams JB, Lowe B. A brief measure for assessing generalized anxiety disorder: the GAD-7. Arch Intern Med. 2006;166:1092-1097.

25. Kroenke K, Spitzer RL, Williams JBW. The PHQ-15: validity of a new measure for evaluating severity of somatic symptoms. Psychosom Med. 2002;64(2):258-266.

26. Hoge CW, McGurk D, Thomas JL, Cox AL, Engel CC, Castro CA. Mild traumatic brain injury in U.S. soldiers returning from Iraq. $N$ Engl J Med. 2008;358(5):453-463.

27. Sha MC, Callahan CM, Counsell SR, Westmoreland GR, Stump TE, Kroenke K. Physical symptoms as a predictor of health care use and mortality among older adults. Am J Med. 2005;118(3):301-306.

28. Brennan KA, Clark CL, Shaver PR. Self-report measurement of adult attachment. In: Simpson JA, Rhodes WS, editors. Attachment Theory and Close Relationships. New York, NY: Guildford; 1988:47-76.

29. Nasreddine ZS, Phillips NA, Bedirian V, et al. The Montreal Cognitive Assessment, MoCA: a brief screening tool for mild cognitive impairment. J Am Geriatr Soc. 2005;53(4):695-699.

30. Tarrants M, Oleen-Burkey M, Castelli-Haley J, Lage MJ. The impact of comorbid depression on adherence to therapy for multiple sclerosis. Mult Scler Int. 2011(2011),271321.

31. DiMatteo MR, Haskard KB, Williams SL. Health beliefs, disease severity, and patient adherence: a meta-analysis. Med Care. 2007;45(6): 521-528.

32. Mackenzie IS, Morant SV, Bloomfield GA, MacDonald TM, O'Riordan J. Incidence and prevalence of multiple sclerosis in the UK 1990-2010: a descriptive study in the General Practice Research Database. J Neurol Neurosurg Psychiatry. 2014;85(1):76-84.
Patient Preference and Adherence

\section{Publish your work in this journal}

Patient Preference and Adherence is an international, peer-reviewed, open access journal that focuses on the growing importance of patient preference and adherence throughout the therapeutic continuum. Patient satisfaction, acceptability, quality of life, compliance, persistence and their role in developing new therapeutic modalities and compounds to optimize

\section{Dovepress}

clinical outcomes for existing disease states are major areas of interest for the journal. This journal has been accepted for indexing on PubMed Central. The manuscript management system is completely online and includes a very quick and fair peer-review system, which is all easy to use. Visit http://www. dovepress.com/testimonials.php to read real quotes from published authors. 